\title{
Uncertainties in the lightest $C P$ even Higgs boson mass prediction in the minimal supersymmetric standard model: fixed order versus effective field theory prediction
}

\author{
B. C. Allanach ${ }^{1, a}$, A. Voigt ${ }^{2, b}$ \\ ${ }^{1}$ DAMTP, CMS, University of Cambridge, Wilberforce Road, Cambridge CB1 3BZ, UK \\ ${ }^{2}$ Institute for Theoretical Particle Physics and Cosmology, RWTH Aachen University, 52074 Aachen, Germany
}

Received: 14 May 2018 / Accepted: 2 July 2018 / Published online: 12 July 2018

(C) The Author(s) 2018

\begin{abstract}
We quantify and examine the uncertainties in predictions of the lightest $C P$ even Higgs boson pole mass $M_{h}$ in the Minimal Supersymmetric Standard Model (MSSM), utilising current spectrum generators and including some threeloop corrections. There are two broadly different approximations being used: effective field theory (EFT) where an effective Standard Model (SM) is used below a supersymmetric mass scale, and a fixed order calculation, where the MSSM is matched to QCD $\times$ QED at the electroweak scale. The uncertainties on the $M_{h}$ prediction in each approach are broken down into logarithmic and finite pieces. The inferred values of the stop mass parameters are sensitively dependent upon the precision of the prediction for $M_{h}$. The fixed order calculation appears to be more accurate below a supersymmetry (SUSY) mass scale of $M_{S} \approx 1.2 \mathrm{TeV}$, whereas above this scale, the EFT calculation is more accurate. We also revisit the range of the lightest stop mass across finetuned parameter space that has an appropriate stable vacuum and is compatible with the lightest $C P$ even Higgs boson $h$ being identified with the one discovered at the ATLAS and CMS experiments in 2012; we achieve a maximum value of $\sim 10^{11} \mathrm{GeV}$.
\end{abstract}

\section{Introduction}

The 2012 discovery at Large Hadron Collider experiments [1, 2] of a resonance that has measured properties compatible

B. C. Allanach: This work has been partially supported by STFC consolidated grant ST/P000681/1.

A. Voigt: A. Voigt acknowledges support by the DFG Research Unit New Physics at the LHC (FOR2239).

a e-mail: B.C.Allanach@damtp.cam.ac.uk

b e-mail: alexander.voigt@physik.rwth-aachen.de with those of a SM Higgs boson, raises some expectations. Should the language of quantum field theory be interpreted correctly by most of the research community, huge corrections to the Higgs boson mass are expected, rendering its measured value [3] of

$M_{h}=125.09 \pm 0.32 \mathrm{GeV}$

untenable unless its value is finely tuned with unrelated contributions cancelling to a suspiciously large degree. This technical hierarchy problem can be solved by new physics that appears around the $\mathrm{TeV}$ scale, the foremost example being $\mathrm{TeV}$ scale supersymmetry. $\mathrm{TeV}$ scale supersymmetry predicts that the masses of new hitherto undiscovered sparticles are not much higher than the $\mathrm{TeV}$ scale. These to date have not been discovered, and the most natural portion of supersymmetry parameter space is being heavily squeezed by experimental constraints.

It is possible that there is some misunderstanding in the way that quantum field theory generates such huge corrections and that the technical hierarchy problem should be taken cum grano salis. It is also possible that supersymmetry is simply a little late to the LHC party, is a little heavier than expected and isn't quite as natural as was originally thought. It is therefore crucial to try to discover superparticles. Within the simplest supersymmetric extension of the $\mathrm{SM}$, the MSSM, there is a lot of parameter space where $h$ appears to be essentially SM like. Its mass, which is $M_{h}=$ $M_{Z} \cos 2 \beta$ at tree level in the decoupling limit $\left(M_{Z}\right.$ being the mass of the $Z$ boson and $\tan \beta=v_{u} / v_{d}$ is the ratio of the two neutral $C P$-even MSSM Higgs field vacuum expectation values (VEVs)), receives large corrections at the loop level. It has been known for some time that the largest corrections to its mass (squared) come from top/stop corrections, which are enhanced by the large value of the top mass [4]: 
$M_{h}^{2}=M_{Z}^{2} \cos ^{2} 2 \beta+\frac{3}{2 \pi^{2}} \frac{m_{t}^{4}}{v^{2}}\left[\ln \frac{M^{2}}{m_{t}^{2}}+\frac{X_{t}^{2}}{M^{2}}-\frac{X_{t}^{4}}{12 M^{4}}\right]$,

where $M=\sqrt{m_{\tilde{t}_{1}} m_{\tilde{t}_{2}}}, m_{\tilde{t}_{i}}$ is the running $i$ th stop mass, $m_{t}$ is the running top mass, $X_{t}=A_{t}-\mu / \tan \beta$ is the running stop mixing parameter and $v \sim 246 \mathrm{GeV}$ is the running SM-like Higgs VEV. Each quantity on the right hand side of Eq. (2) is evaluated at some $\overline{\mathrm{DR}}^{\prime}[5-7]$ renormalisation scale $Q$. The stops play a crucial rôle in bringing the value of $M_{h}$ predicted up to the measured value from the tree-level value. The measured value of $M_{h}$ in Eq. (1) prefers those parts of parameter space that have larger stop masses and/or large mixing between the two stops.

The truncation of perturbation theory at a finite order generates a theoretical uncertainty on the prediction of $M_{h}$. This then leads to an associated uncertainty in the inferred masses and mixings of stops that agree with the experimentally inferred value of $M_{h}$. The allowed range of stop parameter space depends very sensitively on the accuracy of the $M_{h}$ prediction. Equation (2) shows that the stop mass scale depends roughly exponentially upon $M_{h}$ in the high $m_{\tilde{t}_{i}}$ limit. ${ }^{1}$ Achieving the most precise prediction for $M_{h}$ is then of paramount importance. In order to predict $M_{h}$ with higher accuracy and greater precision, higher-order contributions and large log re-summation are required. To date, terms up to two-loop order have been computed in the onshell scheme [8-19] and up to three-loop order in the $\overline{\mathrm{DR}} / \overline{\mathrm{DR}}^{\prime}$ scheme [4, 13-15,20-32].

Currently, ATLAS and CMS perform many different searches for stops. They depend upon various MSSM parameters, but in the more constraining and direct cases, the searches rule out lightest stop masses up to around $1 \mathrm{TeV}$ $[33,34]$. Ideally, it would be useful to determine exactly how heavy the stops might be so that it can be judged how much of the viable parameter space has been excluded and so that one may inform the utility of future higher energy colliders such as the Future Circular Collider (FCC) [35-37]. However, it was shown in Refs. [38-42] that stops far heavier than the $100 \mathrm{TeV}$ putative centre of mass energy of the FCC are compatible with Eq. (1), provided that one is willing to accept the tuning in $v$ implied by the technical hierarchy problem. ${ }^{2} \mathrm{We}$ shall repeat this calculation taking our more precise estimates of the theoretical uncertainties in $M_{h}$ into account.

There are several current publicly available MSSM spectrum calculator computer programs on the market. These calculate the spectrum consistent with weak-scale data on the gauge couplings and the masses of SM states. Each employs

\footnotetext{
1 More precisely, $M_{h}^{2}$ has a logarithmic dependence on $M$ in the large $M$ limit.

2 Large stop masses make the running soft-breaking squared Higgs mass parameters very large, requiring a huge cancellation in the minimisation of the Higgs potential to achieve $v \sim 246 \mathrm{GeV}$.
}

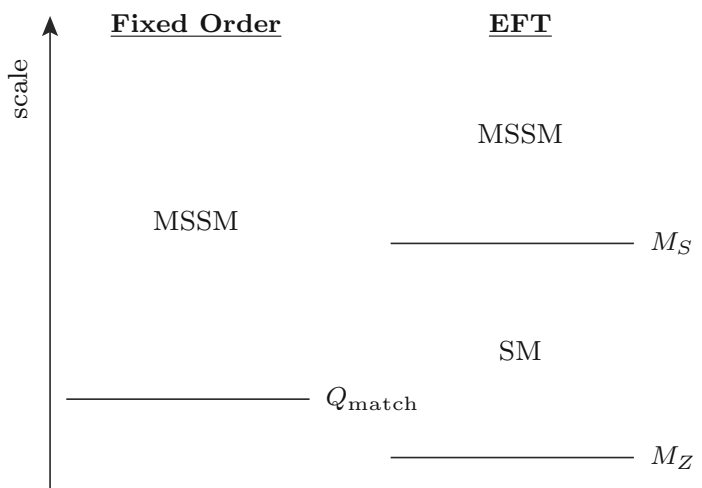

$\mathrm{QCD} \times \mathrm{QED}$

$\mathrm{QCD} \times \mathrm{QED}$

Fig. 1 Schematics of different MSSM approximation schemes: fixed order $\overline{\mathrm{DR}}^{\prime}$ and EFT. In the fixed order $\overline{\mathrm{DR}}^{\prime}$ scheme the MSSM is matched to effective QCD $\times$ QED at the scale $Q_{\text {match }}$. In the EFT scheme the MSSM is matched to an effective SM at the supersymmetric scale $M_{S}$. Horizontal lines show the matching scales

an approximation scheme. The two approximation schemes examined here are called the fixed order $\overline{\mathrm{DR}}^{\prime}$ scheme and the EFT scheme, depicted in Fig. 1. The fixed order $\overline{\mathrm{DR}}^{\prime}$ scheme matches effective $\mathrm{QED} \times \mathrm{QCD}$ to the MSSM at a single scale $Q_{\text {match }}$. The values $Q_{\text {match }}=M_{Z}$ or $Q_{\text {match }}=M_{t}$ are commonly taken, and data on gauge couplings and quark, lepton and electroweak boson masses are input at this scale $Q_{\text {match }}$ (see Ref. [43] for a more detailed description). These couplings are then run using MSSM renormalisation group equations (RGEs) to $M$, where the Higgs potential minimisation conditions are imposed and supersymmetric physical observables including $M_{h}$ are calculated. $M_{h}$ is calculated using the known higher order diagrammatic corrections, up to three loops, of the order $o \in\left\{\alpha_{\mathrm{s}} \alpha_{t}, \alpha_{\mathrm{s}} \alpha_{b}, \alpha_{t}{ }^{2}, \alpha_{t} \alpha_{b}, \alpha_{b}{ }^{2}\right.$, $\left.\alpha_{\tau}^{2}, \alpha_{\mathrm{s}}^{2} \alpha_{t}\right\}$, where $\alpha_{\mathrm{s}}=g_{3}{ }^{2} /(4 \pi), \alpha_{t, b, \tau}=y_{t, b, \tau}^{2} /(4 \pi)$ and $y_{t}, y_{b}, y_{\tau}$ are the top, bottom and tau Yukawa couplings, respectively, and $g_{3}$ is the QCD gauge coupling. These fixed-order corrections include two-loop terms which are proportional to $o \ln ^{2}\left(M_{S} / M_{Z}\right) /(4 \pi)^{2}$ as well as terms of order $o M_{Z}^{2} / M_{S}^{2} /(4 \pi)^{2}$. However, some three-loop terms, for example of order $\left\{\alpha_{t}{ }^{2} \alpha_{s}, \alpha_{t}{ }^{3}\right\} \times \ln ^{3}\left(M_{S} / M_{Z}\right) /(4 \pi)^{3}$, are missed. As $M_{S}$ becomes larger (for example as motivated by the negative results of sparticle searches), such missing logarithmic higher order terms become numerically more important, and missing them will imply a larger uncertainty in the fixed order $\overline{\mathrm{DR}}^{\prime}$ prediction of $M_{h}$. This has motivated the approximation scheme which we call the EFT scheme, where the heavy SUSY particles are decoupled at the SUSY scale $M_{S}$ and the RGEs are used to re-sum the large logarithmic corrections. However, the EFT scheme neglects terms of order $M_{Z}^{2} / M_{S}^{2}$ at the tree level and therefore is less accurate 
the closer $M_{S}$ is to $M_{Z}$. Which scheme is the most accurate for various different physical predictions is not obvious beforehand and depends on the MSSM parameters. It is one of our goals to determine in which domain of $M_{S}$ the fixedorder scheme becomes less accurate than the EFT scheme.

The preceding paragraph has been greatly simplified for clarity of discussion. In the MSSM there are many gauge and Yukawa couplings and one-loop corrections from all of these are included in the fixed order $\overline{\mathrm{DR}}^{\prime}$ calculations. Also, we have used $M_{S}$ as a catch-all supersymmetric scale, but really the individual sparticles contribute to the logarithms and finite terms with their own masses, not with some universal value of $M_{S}$.

The programs used for our $M_{h}$ predictions are the fixed order $\overline{\mathrm{DR}}^{\prime}$ spectrum generators SOFTSUSY 4.1.1 [43,44], FlexibleSUSY 2.1.0 [45,46] and HSSUSY 2.1.0 [46], which uses the EFT approach. We include the three-loop corrections that are available in Himalaya 1.0.1 [47].

In Ref. [48], the hybrid fixed order $\overline{\mathrm{DR}}^{\prime}$ EFT calculation of FeynHiggs $[49,50]$ was compared to the purely EFT calculation of SUSYHD [42]. The observed numerical differences between the (mostly) on-shell hybrid calculation of FeynHiggs and the $\overline{\mathrm{DR}}^{\prime}$ calculation of SUSYHD were found to be mainly caused by renormalisation scheme conversion terms, the treatment of higher-order terms in the determination of the Higgs boson pole mass and the parametrisation of the top Yukawa coupling. When these differences are taken into consideration, excellent agreement was found between the two programs for SUSY scales above $1 \mathrm{TeV}$. This finding confirms that above this scale the terms neglected in the EFT calculation are in fact negligible and the EFT calculation yields an accurate prediction of the Higgs boson mass. Similarly, in Ref. [51] the $\overline{\mathrm{DR}}^{\prime}$ hybrid fixed order/EFT calculation implemented in FlexibleSUSY (denoted as FlexibleEFTHiggs) was compared to the $\overline{\mathrm{DR}}^{\prime}$ fixed order calculation available in FlexibleSUSY. A prescription for an uncertainty estimation of both calculations was given and it was found that (based on that uncertainty estimate) above a few $\mathrm{TeV}$ the hybrid and the pure EFT calculations are more precise than the fixed order $\overline{\mathrm{DR}}^{\prime}$ calculation.

Our work differs from Refs. $[48,51]$ in that we perform a comparison between the $\overline{\mathrm{DR}}^{\prime}$ fixed order and the pure EFT predictions. Our $\overline{\mathrm{DR}}^{\prime}$ fixed order calculation is also a loop higher in order than the previous work. We shall give a prescription for the estimation of the theoretical uncertainties of the two $M_{h}$ predictions in the $\overline{\mathrm{DR}}^{\prime}$ scheme based on the procedures described in Refs. [40,42,51]. Based on our uncertainty estimates we derive an $M_{S}$ region in that scheme, above which the EFT prediction becomes more precise than the fixed order one.

In Sect. 2, we estimate and dissect theoretical uncertainties in state-of-the art predictions of the lightest $C P$ even Higgs boson pole mass in the $\overline{\mathrm{DR}}^{\prime}$ scheme. Then, in Sect. 3, we update the upper bounds on the lightest stop mass from the experimental determination of the Higgs boson mass and from the stability of an appropriate vacuum by our detailed quantification of the theoretical uncertainties and state-ofthe-art calculation of $M_{h}$. We summarise in Sect. 4.

\section{Higgs boson mass prediction uncertainties}

Sources of uncertainty in the $\overline{\mathrm{DR}}^{\prime}$ fixed-order calculation of the lightest $C P$-even Higgs boson pole mass prediction can be divided into two classes:

- Missing higher order contributions to the Higgs self energy and to the electroweak symmetry breaking (EWSB) conditions.

- Missing higher order corrections in the determination of the running $\overline{\mathrm{DR}}^{\prime}$ gauge and Yukawa couplings and the VEVs from experimental quantities.

The prescription presented in Ref. [51] to estimate these missing higher order contributions is sensitive to leading and subleading logarithmic as well as non-logarithmic terms. An analysis of how these different kinds of higher order terms enter the uncertainty estimate can be found in that reference. In the $C P$-conserving MSSM the known two- and three-loop contributions to the $C P$ even Higgs self energy and EWSB conditions are included. The currently unknown (subleading) logarithmic higher order corrections can be estimated by varying the renormalisation scale at which the Higgs boson mass is calculated, $Q_{\text {pole }}$. We estimate this uncertainty as in Ref. [51],

$\Delta M_{h}^{\left(Q_{\text {pole }}\right)}=\max _{Q_{\text {pole }} \in\left[M_{S} / 2,2 M_{S}\right]}\left|M_{h}\left(Q_{\text {pole }}\right)-M_{h}\left(M_{S}\right)\right|$,

where $M_{S}$ is the SUSY scale, usually set to $M_{S}=\sqrt{m_{\tilde{t}_{1}} m_{\tilde{t}_{2}}}$. In Fig. 2 we show this uncertainty as the blue dashed line for a scenario with degenerate $\overrightarrow{\mathrm{DR}}^{\prime}$ mass parameters (aside from the Higgs mass parameters, which are fixed in order to achieve successful EWSB), $\tan \beta=20$ and maximal stop mixing, $X_{t}=-\sqrt{6} M_{S}$. For this scenario $\Delta M_{h}^{\left(Q_{\text {pole }}\right)}$ varies between $0.5-1 \mathrm{GeV}$, depending on the SUSY scale. In Ref. [51] this uncertainty is larger, because the three-loop contribution to the Higgs boson mass was not included. The kink at $M_{S} \approx 700 \mathrm{GeV}$ is due to a switch in the approximation scheme being used in the calculation of the threeloop contribution of Himalaya: the integrands of the threeloop integrals were expanded in different sparticle "mass hierarchies" where different sparticles were approximated as being massless [31]. As $M_{S}$ changes, Himalaya switches from one mass hierarchy to another one that fits better to 


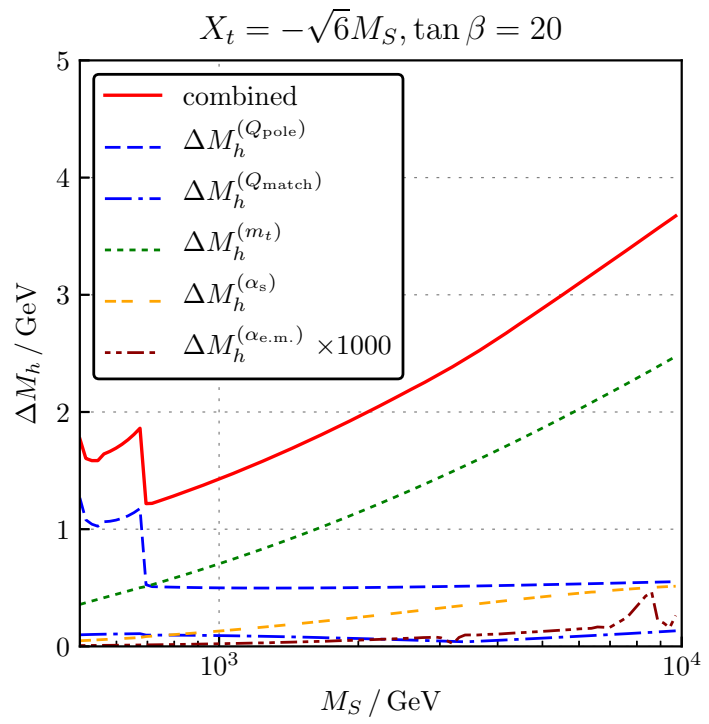

Fig. 2 Individual sources of uncertainty of the three-loop fixed order $\overline{\mathrm{DR}}^{\prime}$ Higgs boson mass prediction of SOFTSUSY

the given parameter point, resulting in the kink. We note that $\Delta M_{h}^{\left(Q_{\text {pole }}\right)}$ is approximately independent of $M_{S}$ as $M_{S}$ becomes large. The dominant $Q_{\text {pole }}$ dependence comes from the first term on the right-hand side of Eq. (2): from the running $Z$ mass, at one-loop order. This will be cancelled by to leading order in $\log \left(Q_{\text {pole }}\right)$ by the one-loop electroweak corrections that are added to $M_{h}$ by the spectrum generators that we employ. However, higher order logarithms (formally at the two-loop order) in the electroweak gauge couplings remain. These remaining pieces have no explicit dependence at leading order on $M=M_{S}$. In the limit of large $M_{S}$, the first term in the square brackets of Eq. (2) contains both a dependence on a large $M_{S}$ and $Q_{\text {pole }}$ through renormalisation of the running top Yukawa coupling $y_{t}=\sqrt{2} m_{t} /(v \sin \beta)$. The $Q_{\text {pole }}$ dependence of leading logarithm terms due to this are cancelled by the explicit two-loop terms of order $\alpha_{t}^{2} /(4 \pi)^{2}$ in the $M_{h}$ calculation that the spectrum generators employ, but higher powers of the logarithms do not cancel. The $Q_{\text {pole }}$ dependence from this term is then formally of three-loop order, but is boosted somewhat by the large value of $y_{t}$. For $\tan \beta=20$ and large $M_{S}$, the $Q_{\text {pole }}$ dependence is small, partly aided by cancellations in the beta function of $y_{t}$. However, for $\tan \beta=5$, as is the case in Ref. [51], for example, one can see an increase in scale uncertainty with a larger $M_{S}$ due to a larger value of $y_{t}$ (and consequently a larger beta function/scale dependence).

The size of the missing (subleading) logarithmic higher order contributions to the running MSSM $\overline{\mathrm{DR}}^{\prime}$ gauge and Yukawa couplings can be estimated in a similar way to that of $Q_{\text {pole }}$ by varying the renormalisation scale $Q_{\text {match }}$, at which the said parameters are determined. We define this uncer- tainty as

$$
\Delta M_{h}^{\left(Q_{\text {match }}\right)}=\max _{Q \in\left[Q_{\text {match }} / 2,2 Q_{\text {match }}\right]}\left|M_{h}(Q)-M_{h}\left(Q_{\text {match }}\right)\right|
$$

where $Q_{\text {match }}$ is the scale at which these parameters are determined, usually set to $M_{Z}$ or $M_{t}$. The uncertainty $\Delta M_{h}^{\left(Q_{\text {match }}\right)}$ is shown as blue dashed-dotted line in Fig. 2 and is below $0.2 \mathrm{GeV}$ for the scenario shown.

Besides the logarithmic higher order corrections there are also 'non-logarithmic' higher corrections, which are important and should be taken into account in any robust uncertainty estimate [51]. We estimate these non-logarithmic corrections by changing the calculation of the running MSSM parameters $m_{t}, \alpha_{\mathrm{s}}$ and $\alpha_{\text {e.m. }}$. by higher orders: the running $\overline{\mathrm{DR}}^{\prime}$ top mass $m_{t}$ is calculated in two different ways, similar to Ref. [51]:

$$
\begin{aligned}
m_{t}^{(1)}= & M_{t}+\widetilde{\Sigma}_{t}^{(1), S}+M_{t}\left[\widetilde{\Sigma}_{t}^{(1), L}+\widetilde{\Sigma}_{t}^{(1), R}\right] \\
& +M_{t}\left[\widetilde{\Sigma}_{t}^{(1), \mathrm{SQCD}}+\widetilde{\Sigma}_{t}^{(2), \mathrm{SQCD}}+\left(\widetilde{\Sigma}_{t}^{\left.(1), \mathrm{SQCD})^{2}\right]}\right.\right.
\end{aligned}
$$

and

$$
\begin{aligned}
m_{t}^{(2)}=M_{t} & +\widetilde{\Sigma}_{t}^{(1), S}+m_{t}\left[\widetilde{\Sigma}_{t}^{(1), L}+\widetilde{\Sigma}_{t}^{(1), R}\right] \\
& +m_{t}\left[\widetilde{\Sigma}_{t}^{(1), \mathrm{SQCD}}+\widetilde{\Sigma}_{t}^{(2), \mathrm{SQCD}}\right],
\end{aligned}
$$

where $M_{t}$ denotes the top pole mass, $\widetilde{\Sigma}_{t}^{(1), S}, \widetilde{\Sigma}_{t}^{(1), L}$ and $\widetilde{\Sigma}_{t}^{(1), R}$ denote the scalar, left-handed and right-handed part of the one-loop top self energy without SUSY-QCD contributions and $\widetilde{\Sigma}_{t}^{(1,2), S Q C D}$ denote the one-loop and two-loop SUSY-QCD contributions from Refs. [52-54]. ${ }^{3}$ Note, that in Ref. [51] the two-loop SM-QCD contribution has been used on the right hand side of Eqs. (5) and (6), while here we use the full two-loop SUSY-QCD contribution of $\mathcal{O}\left(\alpha_{s}^{2}\right)$. Since the latter is significantly larger, the difference between $m_{t}^{(1)}$ and $m_{t}^{(2)}$ is larger in our case. Equations (5) and (6) are equivalent at $\mathcal{O}\left(\alpha_{\mathrm{s}}{ }^{2}\right)$, but differ at $\mathcal{O}\left(\alpha_{\text {e.m. }} \alpha_{\mathrm{s}}\right)$, for example. Since the difference contains both logarithmic and non-logarithmic terms, it can be used as an uncertainty estimate. Similar to Ref. [51] we define

$\Delta M_{h}^{\left(m_{t}\right)}=\left|M_{h}\left(m_{t}^{(1)}\right)-M_{h}\left(m_{t}^{(2)}\right)\right|$.

In Ref. [51] four different top mass calculations are combined, whilst we combine only two. The size of $\Delta M_{h}^{\left(m_{t}\right)}$ is shown in Fig. 2 as a green dotted line. Since $\Delta M_{h}^{\left(m_{t}\right)}$ contains terms of the form $\log \left(m_{t} / m_{\tilde{t}_{i}}\right)$, the uncertainty increases $\log$ arithmically with the SUSY scale. It therefore serves as an

${ }_{3}^{3}$ Note that the terms involving square brackets differ in Eqs. (5) and (6). 
estimate of both (leading) logarithmic and non-logarithmic higher order corrections and is a reasonable measure to express the fact that the fixed-order calculation suffers from a large theoretical uncertainty for multi-TeV stop masses.

We estimate the effect of unknown higher order logarithmic and non-logarithmic threshold corrections to $\alpha_{\mathrm{s}}$ and $\alpha_{\mathrm{e} . \mathrm{m}}$. in a similar way to our approach for estimating $\Delta M_{h}^{\left(m_{t}\right)}$ :

$\alpha_{\mathrm{s}}^{(1)}=\frac{\alpha_{\mathrm{s}}{ }^{\mathrm{SM}(5)}}{1-\Delta^{(1)} \alpha_{\mathrm{s}}-\Delta^{(2)} \alpha_{\mathrm{s}}}$,

$\alpha_{\mathrm{s}}^{(2)}=\alpha_{\mathrm{s}}{ }^{\mathrm{SM}(5)}\left[1+\Delta^{(1)} \alpha_{\mathrm{s}}+\left(\Delta^{(1)} \alpha_{\mathrm{s}}\right)^{2}+\Delta^{(2)} \alpha_{\mathrm{s}}\right]$,

and

$$
\begin{aligned}
\alpha_{\mathrm{e} . \mathrm{m} .}^{(1)}= & \frac{\alpha_{\mathrm{e} . \mathrm{m} .}^{\mathrm{SM}(5)}}{1-\Delta^{(1)} \alpha_{\mathrm{e} . \mathrm{m} .}-\Delta^{(2)} \alpha_{\mathrm{e} . \mathrm{m} .}}, \\
\alpha_{\mathrm{e} . \mathrm{m} .}^{(2)}= & \alpha_{\mathrm{e} . \mathrm{m} .}^{\mathrm{SM}(5)}\left[1+\Delta^{(1)} \alpha_{\mathrm{e} . \mathrm{m} .}+\left(\Delta^{(1)} \alpha_{\mathrm{e} . \mathrm{m} .}\right)^{2}\right. \\
& \left.+\Delta^{(2)} \alpha_{\mathrm{e} . \mathrm{m} .}\right]
\end{aligned}
$$

and take the difference as an uncertainty estimate,

$$
\begin{gathered}
\Delta M_{h}^{\left(\alpha_{\mathrm{s}}\right)}=\left|M_{h}\left(\alpha_{\mathrm{s}}^{(1)}\right)-M_{h}\left(\alpha_{\mathrm{s}}^{(2)}\right)\right|, \\
\Delta M_{h}^{\left(\alpha_{\text {e.m. }}\right)}=\left|M_{h}\left(\alpha_{\text {e.m. }}^{(1)}\right)-M_{h}\left(\alpha_{\text {e.m. }}^{(2)}\right)\right| .
\end{gathered}
$$

Note that the uncertainties estimated by $\Delta M_{h}^{\left(\alpha_{\mathrm{s}}\right)}$ and $\Delta M_{h}^{\left(\alpha_{\mathrm{e} . \mathrm{m} .}\right)}$ were not included in Ref. [51]. Their respective sizes are shown in Fig. 2 as yellow dashed and brown double-dotted lines, respectively. Due to the logarithmic contributions of the form $\log \left(m_{t} / m_{\tilde{t}_{i}}\right)$ to the threshold corrections of $\alpha_{\mathrm{S}}$ and $\alpha_{\text {e.m. }}$, the two uncertainties increase logarithmically with the SUSY scale. However, since $\alpha_{\text {e.m. }}$ is very small, the uncertainty $\Delta M_{h}^{\left(\alpha_{\mathrm{e} . \mathrm{m} .}\right)}$ is negligible. The magnitude of $\Delta M_{h}^{\left(\alpha_{s}\right)}$ can be around $20 \%$ of $\Delta M_{h}^{\left(m_{t}\right)}$ for large $M_{S}$.

There are some inter-dependencies between the different sources of uncertainty and it is practically impossible to exactly take these into account unless the higher order corrections are explicitly calculated. However, the quantification of theoretical uncertainties is an inexact pursuit and it serves us well enough to combine the different sources of uncertainty linearly

$$
\begin{aligned}
\Delta M_{h}^{(\mathrm{SS}+\mathrm{H})}= & \Delta M_{h}^{\left(Q_{\mathrm{pole}}\right)}+\Delta M_{h}^{\left(Q_{\mathrm{match}}\right)}+\Delta M_{h}^{\left(m_{t}\right)} \\
& +\Delta M_{h}^{\left(\alpha_{\mathrm{s}}\right)}+\Delta M_{h}^{\left(\alpha_{\mathrm{e} . \mathrm{m} .}\right)}
\end{aligned}
$$

in order to have some kind of reasonable estimate of the total level of theoretical uncertainty in the prediction. The combination $\Delta M_{h}^{(\mathrm{SS}+\mathrm{H})}$ is shown in Fig. 2 as a red solid line. As expected, due to logarithmic contributions of the form $\log \left(m_{t} / m_{\tilde{t}_{i}}\right)$, the combined uncertainty of the fixed-order calculation of SOFTSUSY increases with the SUSY scale and reaches $\Delta M_{h}^{(\mathrm{SS}+\mathrm{H})} \sim 4 \mathrm{GeV}$ for $M_{S} \sim 10 \mathrm{TeV}$. The size of the individual uncertainties show that the prescription proposed in Ref. [51] is reasonable, because the additional uncertainties $\Delta M_{h}^{\left(Q_{\text {match }}\right)}, \Delta M_{h}^{\left(\alpha_{s}\right)}$ and $\Delta M_{h}^{\left(\alpha_{\text {e.m. }}\right)}$ that we have introduced here are small. However, compared to the combined uncertainty estimate of Ref. [51] our combined uncertainty is smaller by about $40 \%$ for SUSY scales of around $M_{S} \sim 1 \mathrm{TeV}$ and about $25 \%$ smaller for $M_{S} \sim 10 \mathrm{TeV}$. The main reasons are the reduced scale uncertainty $\Delta M_{h}^{\left(Q_{\text {pole }}\right)}$ due to the three-loop Higgs boson mass corrections that are included here and our different definition of $\Delta M_{h}^{\left(m_{t}\right)}$.

In the following we compare the fixed-order Higgs boson mass prediction for this scenario to the pure EFT calculation of HSSUSY [46]. HSSUSY is a spectrum generator from the FlexibleSUSY package, which implements the highscale SUSY scenario, where the quartic SM Higgs coupling $\lambda\left(M_{S}\right)$ is predicted from matching to the MSSM at a high SUSY scale $M_{S}$. It provides a prediction of the Higgs pole mass in the MSSM in the pure EFT limit, $v^{2} \ll M_{S}^{2}$, up to the two-loop level $\mathcal{O}\left(\alpha_{\mathrm{s}}\left(\alpha_{t}+\alpha_{b}\right)+\left(\alpha_{t}+\alpha_{b}\right)^{2}+\alpha_{\tau} \alpha_{b}+\alpha_{\tau}^{2}\right)$ [40,55-57], including next-to-next-to-leading-log (NNLL) re-summation $[58,59]$. Additional pure SM three- and fourloop corrections [60-65] can be taken into account on demand.

To estimate the Higgs boson mass uncertainty of HSSUSY we use the procedure developed in Ref. [66], which is an extension of the methods used in Refs. [40,42]. The sources of uncertainty of HSSUSY are divided into the following three categories:

- SM uncertainty from missing higher order corrections in the determination of the running SM $\overline{\mathrm{MS}}$ parameters

- EFT uncertainty from neglecting terms of order $\mathcal{O}\left(v^{2} / M_{S}^{2}\right)$

- SUSY uncertainty from missing higher order contributions from SUSY particles

As in the fixed order $\overline{\mathrm{DR}}^{\prime}$ calculation, we divide the SM uncertainty into a logarithmic and non-logarithmic part. However, since large logarithmic corrections to the Higgs mass are re-summed in the EFT calculation, for the 'logarithmic part', we refer specifically to smaller logarithms of the form $\ln \left(Q_{\text {match }} / m_{\tilde{t}_{1}}\right)$ or $\ln \left(Q_{\text {pole }} / M_{t}\right)$. These small logarithmic higher order corrections are estimated by varying the renormalisation scale $Q_{\text {pole }}$, at which the Higgs boson mass is calculated in the effective SM:

$$
\Delta M_{h}^{\left(Q_{\text {pole }}\right)}=\max _{Q_{\text {pole }} \in\left[M_{t} / 2,2 M_{t}\right]}\left|M_{h}\left(Q_{\text {pole }}\right)-M_{h}\left(M_{t}\right)\right| .
$$

The non-logarithmic part is estimated by switching the threeloop QCD contributions $[60,61]$ on or off in the extraction of the running SM top Yukawa coupling from the top pole mass, 
$\Delta M_{h}^{\left(y_{t}^{\mathrm{SM}}\right)}=\left|M_{h}\left(y_{t}^{\mathrm{SM}, 2 \ell}\left(M_{Z}\right)\right)-M_{h}\left(y_{t}^{\mathrm{SM}, 3 \ell}\left(M_{Z}\right)\right)\right|$.

Although this difference is sensitive to non-logarithmic higher order contributions to the Higgs boson mass, it shows an additional dependence on the separation of the electroweak scale and the SUSY scale (as was observed in Refs. [42,67] and shown in the green dotted line of Fig. 3). The main reason for this dependence is that the running top Yukawa coupling (the largest dimensionless parameter in the MSSM) enters the RGEs of the other MSSM parameters, thus affecting their running below $M_{S}$. The effect is stronger for more separated scales.

The EFT uncertainty is estimated by Ref. [42] by multiplying the one-loop contribution of each individual SUSY particle to the quartic Higgs coupling $\lambda\left(M_{S}\right)$ at the SUSY scale by the factor $\left(1+v^{2} / M_{S}^{2}\right)$. We use the resulting change in the Higgs boson pole mass prediction as an estimate for the EFT uncertainty,

$\Delta M_{h}^{\left(v^{2} / M_{S}^{2}\right)}=\left|M_{h}-M_{h}\left(v^{2} / M_{S}^{2}\right)\right|$,

where $M_{h}\left(v^{2} / M_{S}^{2}\right)$ is the predicted Higgs boson mass with the additional $v^{2} / M_{S}^{2}$ terms. In Ref. [57] it was shown that this uncertainty estimate is very conservative.

The SUSY uncertainty is also divided into a logarithmic and a 'non-logarithmic' part. We estimate the (leading) logarithmic part again by varying the scale $Q_{\text {match }}$, at which the matching of the MSSM to the effective SM is performed, similar to Ref. [42],

$\Delta M_{h}^{\left(Q_{\text {match }}\right)}=\max _{Q \in\left[M_{S} / 2,2 M_{S}\right]}\left|M_{h}(Q)-M_{h}\left(M_{S}\right)\right|$.

Like $\Delta M_{h}^{\left(y_{t}^{\mathrm{SM}}\right)}, \Delta M_{h}^{\left(Q_{\text {match }}\right)}$ also shows an additional dependence on the separation of the electroweak scale and the SUSY scale due to the dependence of the RGEs on the running parameters. The non-logarithmic part is estimated by re-parametrising the threshold correction for $\lambda\left(M_{S}\right)$ in terms of the MSSM top Yukawa coupling at the SUSY scale, $y_{t}{ }^{\operatorname{MSSM}}\left(M_{S}\right)$, and we take the resulting shift in the Higgs boson mass as an estimate for the uncertainty

$\Delta M_{h}^{\left(y_{t}^{\mathrm{MSSM}}\right)}=\left|M_{h}-M_{h}\left(y_{t}^{\mathrm{MSSM}}\left(M_{S}\right)\right)\right|$.

A similar uncertainty was defined in Ref. [51], where the loop order of the calculation of $y_{t}^{\mathrm{MSSM}}\left(M_{S}\right)$ was switched between tree- and one-loop level. Our uncertainty $\Delta M_{h}^{\left(y_{t}^{\mathrm{MSSM}}\right)}$ is significantly smaller than the one used in Ref. [51], because we are working at one loop higher order and the uncertainty there contains large two-loop next-to-leading logarithms (see the discussion in Ref. [46]).

Analogously to our procedure with the fixed order $\overline{\mathrm{DR}}^{\prime}$ calculation, we combine all individual HSSUSY uncertainties

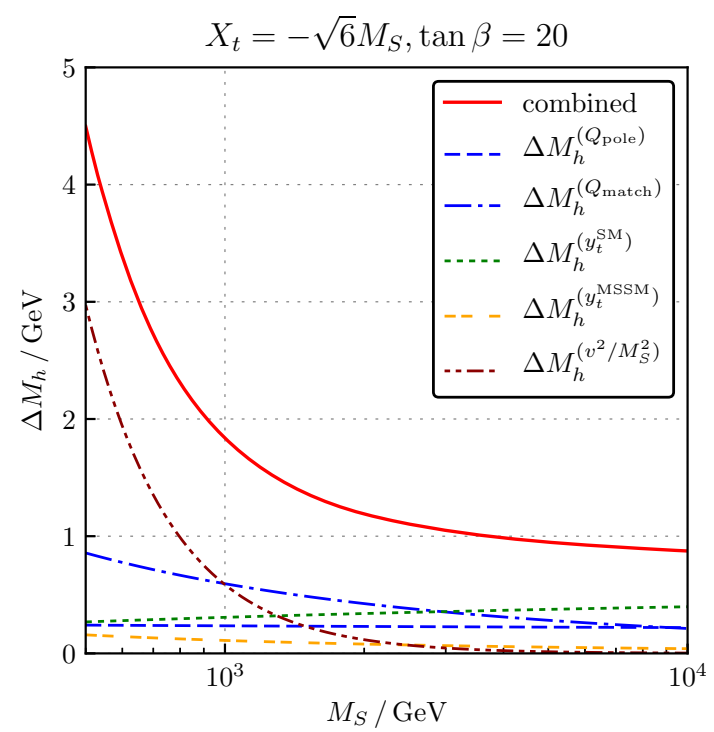

Fig. 3 Individual sources of uncertainty of the two-loop EFT Higgs boson mass prediction of HSSUSY

linearly,

$$
\begin{aligned}
\Delta M_{h}^{(\mathrm{HSSUSY})}= & \Delta M_{h}^{\left(Q_{\text {pole }}\right)}+\Delta M_{h}^{\left(Q_{\text {match }}\right)}+\Delta M_{h}^{\left(y_{t}^{\mathrm{SM}}\right)} \\
& +\Delta M_{h}^{\left(y_{t}^{\mathrm{MSSM}}\right)}+\Delta M_{h}^{\left(v^{2} / M_{S}^{2}\right)}
\end{aligned}
$$

Figure 3 shows the individual uncertainties of HSSUSY from these three categories. For low SUSY scales, $M_{S} \lesssim$ $1 \mathrm{TeV}$, the combined uncertainty estimate of HSSUSY, $\Delta M_{h}^{\text {(HSSUSY) }}$, (red solid line) is dominated by the EFT uncertainty $\Delta M_{h}^{\left(v^{2} / M_{S}^{2}\right)}$ (brown dashed-double-dotted line) due to the fact that the neglected terms of $\mathcal{O}\left(v^{2} / M_{S}^{2}\right)$ are not negligible in this region. For $M_{S} \gtrsim 2 \mathrm{TeV}$ the EFT uncertainty drops below $0.1 \mathrm{GeV}$ and the remaining sources dominate. For even higher scales of $M_{S} \gtrsim 10 \mathrm{TeV}$, the two components of the SUSY uncertainty, $\Delta M_{h}^{\left(Q_{\text {match }}\right)}$ and $\Delta M_{h}^{\left(y_{t}^{\mathrm{MSSM}}\right)}$, become smaller because the dimensionless running couplings become smaller at higher SUSY scales in this scenario. For high scales of $M_{S} \gtrsim 10 \mathrm{TeV}$ the combined uncertainty is dominated by the SM uncertainty, in particular by the uncertainty $\Delta M_{h}^{\left(y_{t}^{\mathrm{SM}}\right)}$ in the extraction of the running SM top Yukawa coupling at the electroweak scale, which remains at $\Delta M_{h}^{\left(y_{t}^{\mathrm{SM}}\right)} \sim 0.5 \mathrm{GeV}$.

In Fig. 4 the $M_{h}$ prediction in the fixed-order and the EFT approximation schemes are shown, together with their uncertainties. ${ }^{4}$ We see from the figure that the allowed $M_{S}$ range depends sensitively on the approximation scheme: 1.3-3.0 TeV for fixed-order and 2.5-4.6 TeV for EFT. The

\footnotetext{
4 There are small differences in the calculations of SOFTSUSY and of FlexibleSUSY producing their $M_{h}$ predictions: for example, the two-loop calculations of the electroweak corrections differ.
} 


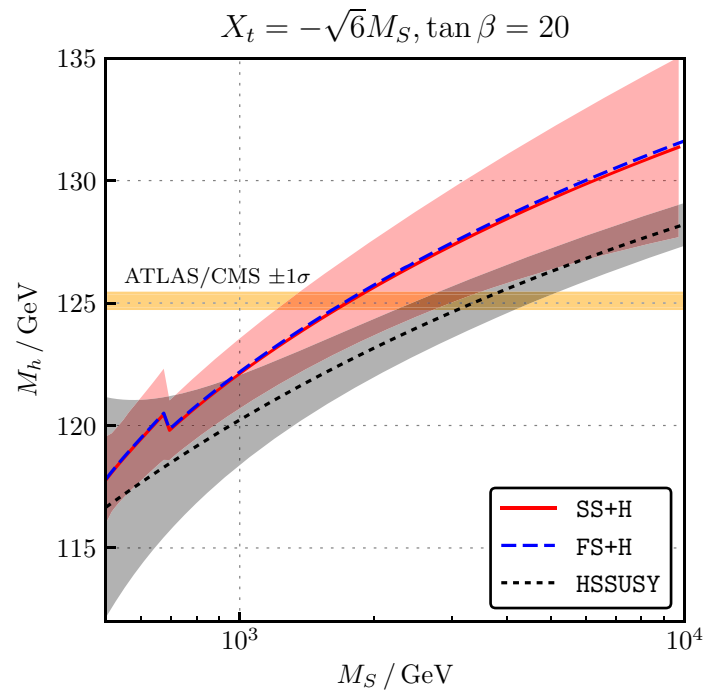

Fig. 4 Higgs boson mass predictions at fixed three-loop order with SOFTSUSY (red solid line) and FlexibleSUSY (blue dashed line) and in the EFT (black dashed line). The coloured regions show the estimated composite theoretical uncertainties in the different predictions of $M_{h}$. The FlexibleSUSY uncertainty is very similar to the SOFTSUSY one, and so is not shown for reasons of clarity. The orange band shows the experimentally measured Higgs boson mass with the experimental uncertainty

Higgs mass increases as a function of the SUSY scale due to the logarithmic enhancement from heavy SUSY particles. As discussed above, the combined uncertainty of the fixed-order calculations (red band) tends to increase with increasing $M_{S}$, while the uncertainty of the EFT calculation (grey band) decreases. The point where the fixed-order and the EFT calculation have the same estimated uncertainty is $M_{S}^{\text {equal }}=1.2 \mathrm{TeV}$. To improve the prediction near this point, a "hybrid" calculation should be used, where the large logarithms are re-summed and $\mathcal{O}\left(v^{2} / M_{S}^{2}\right)$ terms are included $[46,48,50,51,57,68,69]$.

\section{Upper bound on the lightest stop mass}

The logarithmic enhancement of the loop corrections to the Higgs boson mass from heavy stops suggests that there is an upper limit on the mass of the lightest stop from the requirement of predicting $M_{h}=125.09 \mathrm{GeV}$ and a stable and appropriate (i.e. colour and charge preserving) vacuum. As was already shown in Ref. [40], the maximum lightest stop mass is around $10^{11} \mathrm{GeV}$. At very large stop masses, EWSB is fine-tuned, despite the fact that the Higgs mass in our spectrum generators comes out to be small. This is because the generators implicitly tune parameters in order to obtain the measured central value of the $Z$ boson mass $M_{Z}=91.1876$ $\mathrm{GeV}$ (or equivalently, the inferred value of $v \sim 246 \mathrm{GeV}$ ).
We see this in the MSSM EWSB equation [70] which predicts $M_{Z}$ :

$$
\frac{M_{Z}^{2}}{2}=\frac{m_{\bar{H}_{1}}^{2}(Q)-m_{\bar{H}_{2}}^{2}(Q) \tan ^{2} \beta(Q)}{\tan ^{2} \beta(Q)-1}-\frac{1}{2} \Re \Pi_{Z Z}^{T}(Q)-\mu^{2}
$$

where $m_{\bar{H}_{i}}^{2}=m_{H_{i}}^{2}-t_{i} / v_{i}, \Re \Pi_{Z Z}^{T}(Q)$ is the $Z$ self-energy and $t_{i}$ are the tadpole contributions from loops. $Q$ is the scale at which EWSB is calculated: usually around the TeV scale and $v_{i}$ are the two Higgs VEVs of the $C P$ even electrically neutral MSSM Higgs fields. When the stop masses are huge, they contribute to huge values of $m_{\bar{H}_{i}}^{2}$ through the MSSM RGEs, which at one loop order are [71]:

$$
\begin{aligned}
\frac{1}{\kappa} \frac{d m_{H_{1}}^{2}}{d t}= & 6 y_{b}^{2}\left(m_{H_{1}}^{2}+m_{\tilde{Q}_{3}}^{2}+m_{\tilde{d}_{3}}^{2}+A_{b}^{2}\right) \\
& -6 g_{2}^{2} M_{2}^{2}-\frac{6}{5} g_{1}^{2} M_{1}^{2}+\frac{3}{5} g_{1}^{2}\left[m_{H_{2}}^{2}-m_{H_{1}}^{2}\right. \\
& \left.+\operatorname{Tr}\left(m_{\tilde{Q}}^{2}-m_{\tilde{L}}^{2}-2 m_{\tilde{u}}^{2}+m_{\tilde{d}}^{2}+m_{\tilde{e}}^{2}\right)\right], \\
\frac{1}{\kappa} \frac{d m_{H_{2}}^{2}}{d t}= & 6 y_{t}^{2}\left(m_{H_{2}}^{2}+m_{\tilde{Q}_{3}}^{2}+m_{\tilde{u}_{3}}^{2}+A_{t}^{2}\right) \\
& -6 g_{2}^{2} M_{2}^{2}-\frac{6}{5} g_{1}^{2} M_{1}^{2}+\frac{3}{5} g_{1}^{2}\left[m_{H_{2}}^{2}-m_{H_{1}}^{2}\right. \\
& \left.+\operatorname{Tr}\left(m_{\tilde{Q}}^{2}-m_{\tilde{L}}^{2}-2 m_{\tilde{u}}^{2}+m_{\tilde{d}}^{2}+m_{\tilde{e}}^{2}\right)\right],
\end{aligned}
$$

where $t$ is the natural logarithm of the renormalisation scale and $M_{i}, m_{i}$ are soft supersymmetry breaking mass parameters of order $M_{S}$, as defined in Ref. [71]. In order for the left-hand side of Eq. (21) to obtain the experimental value, the first and the last term must cancel to a very large degree. There is no fundamental reason why this is the case and the terms must be tuned.

In practice, HSSUSY inverts the Higgs minimisation equations, taking $\mu\left(M_{S}\right)$ and the value of the $C P$-odd Higgs boson mass $m_{A}\left(M_{S}\right)$ as input values. In this scheme, $m_{\bar{H}_{i}}^{2}$ are implicitly tuned in order to give $M_{Z}^{2}$ at the experimental central value. Once this single tuning has been achieved, there are no more large corrections to $M_{h}$ from heavy sparticles: they are all proportional to $M_{Z} \propto v$, which has already been tuned.

We estimate the upper bound on the lightest $\overline{\mathrm{DR}}^{\prime}$ stop mass $m_{\tilde{t}_{1}}$ in Fig. 5 by scanning over $M_{S}$ and the relative $\overline{\mathrm{DR}}^{\prime}$ stop mixing parameter $X_{t} / M_{S}$ in a scenario with degenerate SUSY breaking mass parameters (except for $m_{H_{i}}^{2}$ ) set equal to $M_{S}, \mu\left(M_{S}\right)=m_{A}\left(M_{S}\right)=M_{S}$ and $\tan \beta=1$ to make the tree-level Higgs mass vanish. This should then be the limiting case where we require the largest correction from stops in order to predict $M_{h}$ in the correct range to satisfy the experimental measurement. The Higgs boson mass has been calculated with the pure EFT calculation HSSUSY, because it has a smaller uncertainty than the fixed-order calculations in the 


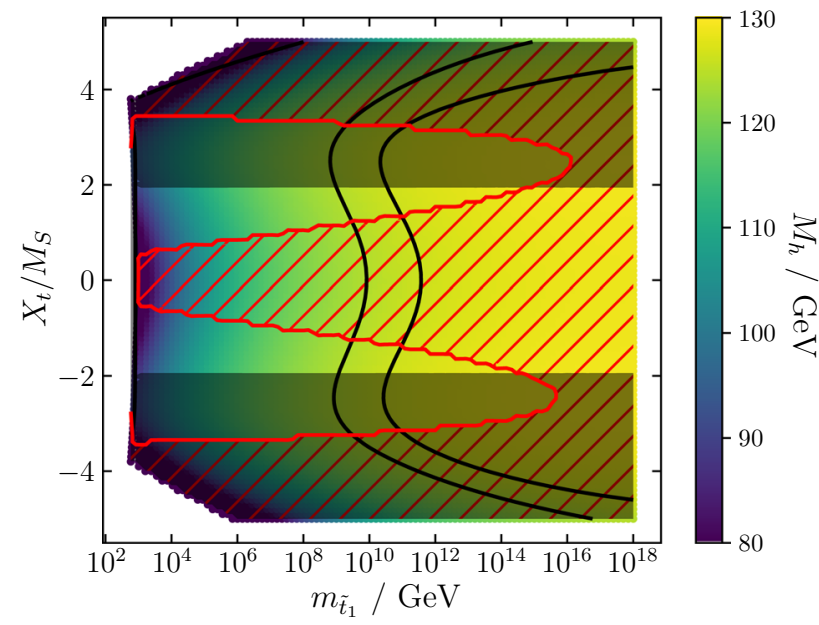

Fig. 5 Higgs boson mass prediction with the EFT calculation HSSUSY as a function of the lightest $\overline{\mathrm{DR}}^{\prime}$ stop mass and the $\overline{\mathrm{DR}}^{\prime}$ stop mixing parameter for degenerate SUSY mass parameters and $\tan \beta\left(M_{S}\right)=1$. The solid lines show the central value of $M_{h}$ according to Eq. (1) plus or minus the theoretical uncertainty. The dark regions at the top and bottom of the plot display regions of parameter space which have charge and colour breaking minima. Hatched regions have $\lambda\left(M_{S}\right)<0$

limit of large stop masses. The black lines show the contours of $M_{h}=125.09 \mathrm{GeV} \pm \Delta M_{h}^{\text {(HSSUSY) }}$, where $\Delta M_{h}^{\text {(HSSUSY) }}$ is the estimate of the theory uncertainty from HSSUSY, as described in Sect. 2. In the red hatched region the quartic Higgs coupling is negative at the SUSY scale, $\lambda\left(M_{S}\right)<0$, pointing to a potentially unstable electroweak vacuum. ${ }^{5,6}$ In order to tell whether a point with $\lambda\left(M_{S}\right)<0$ really is excluded, one should examine the full MSSM scalar potential. We consider this to be beyond the scope of the present work, and so for now, we simply leave it as a point of interest. We also display regions which are excluded because they would lead to charge and colour breaking minima which are deeper than the electroweak vacuum, outside the region [40]

$\frac{X_{t}^{2}}{m_{\tilde{Q}_{3}} m_{\tilde{u}_{3}}}<\left(4-\frac{1}{\sin ^{2} \beta}\right)\left(\frac{m_{\tilde{Q}_{3}}}{m_{\tilde{u}_{3}}}+\frac{m_{\tilde{u}_{3}}}{m_{\tilde{Q}_{3}}}\right)$.

Applying Eq. (24) at $Q=M_{S}$ with our boundary conditions on the quantities within it leads to

$-2<X_{t} / M_{S}<2$

\footnotetext{
$\overline{5 \text { Around } X_{t}} \approx 0$ the Higgsinos and electroweak gauginos give the dominant negative contribution to $\lambda\left(M_{S}\right)$ for $\tan \beta=1$. For slightly larger values of $X_{t}$ the stop contributions become dominant, leading to a positive $\lambda\left(M_{S}\right)$. For large stop mixing, the stop contribution becomes negative as well, driving $\lambda\left(M_{S}\right)<0$ again.

${ }^{6}$ For slightly larger values of $\tan \beta$ the region around $X_{t} \approx 0$ becomes allowed. However, with larger $\tan \beta$ the tree-level Higgs boson mass rapidly increases, which leads to a significantly lower bound on the lightest stop mass.
}

which corresponds to the non-darkened region in the horizontal middle band of Fig. 2. From regions with a stable electroweak vacuum based on the criterion in Eq. (25) and where Eq. (1) is satisfied including the theoretical uncertainty, we estimate an upper bound of $m_{\tilde{t}_{1}}<3.7 \times 10^{11} \mathrm{GeV}$.

\section{Summary}

By using the state-of-the-art EFT Higgs boson mass prediction of HSSUSY we derived an estimate for the upper bound of the lightest running stop mass that is compatible with the measured value of the Higgs boson mass of $M_{h}=125.09$ $\mathrm{GeV}$, taking into account the uncertainty estimate of the EFT calculation. Our estimate for the range of the lightest stop mass is

$m_{\tilde{t}_{1}}<3.7 \times 10^{11} \mathrm{GeV}$,

provided the sparticle spectrum is not split so that some sparticles are much lighter than $m_{\tilde{t}_{1}}$, as this would invalidate the assumptions implicit within the EFT calculation that all sparticles are around $M_{S}$. Our more precise estimate of theoretical uncertainties in the prediction of $M_{h}$ does not qualitatively change the conclusion of the previous study in Ref. [40]. Unfortunately, such a bound is very much higher than the potential energies of conceivable terrestrial particle accelerators.

We also compared the precision of the Higgs boson mass predictions of the state-of-the-art $\overline{\mathrm{DR}}^{\prime}$ fixed-order and EFT spectrum generators SOFTSUSY, FlexibleSUSY and HSSUSY in the MSSM. We estimated the uncertainties of the Higgs boson mass of the fixed-order and the EFT calculation by considering unknown logarithmic and nonlogarithmic higher-order corrections. As part of our work, we have provided a scheme to estimate the theoretical uncertainties in fixed-order $\overline{\mathrm{DR}}^{\prime}$ calculations, based on the prescription used in Ref. [51]. Our prescription is an extension of Ref. [51], which takes further sources of uncertainty into account. By comparing the precision of the predictions of the two methods, we concluded that for SUSY masses below $M_{S}^{\text {equal }}=1.2 \mathrm{TeV}$, the fixed-order calculation is more precise, while above that scale the EFT method is more precise. To estimate this scale, we took the maximal mixing case where all soft supersymmetry breaking masses are set to be degenerate at $M_{S}$ (except for $m_{H_{i}}$, which are fixed in order to achieve successful EWSB) and where $\tan \beta=20$. The precise value of $M_{S}^{\text {equal }}$ will change depending upon the scenario and can vary between $M_{S}=1.0 \mathrm{TeV}$ and $1.3 \mathrm{TeV}$ for min$\mathrm{imal} / \mathrm{maximal}$ stop mixing and small/large values of $\tan \beta$. However, once one imposes the experimental measurement upon $M_{h}, M_{S} \geq 1.3 \mathrm{TeV}$ according to the fixed-order calcu- 
lation $^{7}$ and $2 \mathrm{TeV}$ according to the EFT calculation, as Fig. 4 shows. For $M_{S} \geq 1.3 \mathrm{TeV}$, the EFT has smaller uncertainties and so one is likely to be in a régime where $M_{h}$ is better approximated by EFT methods. It is unclear as yet, however, whether details of the MSSM spectrum other than $M_{h}$ are better approximated by EFT methods. One question which we have not addressed is: which approximation scheme (fixed order $\overline{\mathrm{DR}}^{\prime}$ or EFT) is more accurate when there is a hierarchical sparticle spectrum? It is quite possible, for example, that the stops are heavy but several of the other MSSM sparticles are significantly lighter. For such scenarios the precision of the fixed order $\overrightarrow{\mathrm{DR}}^{\prime}$ calculation would have to be compared with the precision of an appropriate EFT that contains the light sparticles. We leave such a study for future work.

Acknowledgements We would like to thank the KUTS series of workshops, and LPTHE Paris for hospitality extended during the commencement of this work. A.V. would like to thank Emanuele Bagnaschi, Pietro Slavich and Georg Weiglein for many helpful discussions on the Higgs boson mass uncertainty estimate and Emanuele Bagnaschi for providing the two- and three-loop shifts to re-parametrise the quartic Higgs coupling in terms of the MSSM top Yukawa coupling. We thank Pietro Slavich for detailed comments on the manuscript. B.C.A. would like to thank the Cambridge SUSY Working Group for helpful discussions.

Open Access This article is distributed under the terms of the Creative Commons Attribution 4.0 International License (http://creativecomm ons.org/licenses/by/4.0/), which permits unrestricted use, distribution, and reproduction in any medium, provided you give appropriate credit to the original author(s) and the source, provide a link to the Creative Commons license, and indicate if changes were made. Funded by SCOAP ${ }^{3}$.

\section{References}

1. G. Aad et al., Phys. Lett. B 716, 1 (2012). https://doi.org/10.1016/ j.physletb.2012.08.020

2. S. Chatrchyan et al., Phys. Lett. B 716, 30 (2012). https://doi.org/ 10.1016/j.physletb.2012.08.021

3. G. Aad et al., Phys. Rev. Lett. 114, 191803 (2015). https://doi.org/ 10.1103/PhysRevLett.114.191803

4. B.C. Allanach, A. Djouadi, J.L. Kneur, W. Porod, P. Slavich, JHEP 09, 044 (2004). https://doi.org/10.1088/1126-6708/2004/09/044

5. W. Siegel, Phys. Lett. 84B, 193 (1979). https://doi.org/10.1016/ 0370-2693(79)90282-X

6. D.M. Capper, D.R.T. Jones, P. van Nieuwenhuizen, Nucl. Phys. B 167, 479 (1980). https://doi.org/10.1016/0550-3213(80)90244-8

7. I. Jack, D.R.T. Jones, S.P. Martin, M.T. Vaughn, Y. Yamada, Phys. Rev. D 50, R5481 (1994). https://doi.org/10.1103/PhysRevD.50. R5481

8. R. Hempfling, A.H. Hoang, Phys. Lett. B 331, 99 (1994). https:// doi.org/10.1016/0370-2693(94)90948-2

9. S. Heinemeyer, W. Hollik, G. Weiglein, Phys. Lett. B 440, 296 (1998). https://doi.org/10.1016/S0370-2693(98)01116-2

10. S. Heinemeyer, W. Hollik, G. Weiglein, Phys. Rev. D 58, 091701 (1998). https://doi.org/10.1103/PhysRevD.58.091701

$\overline{7}$ For a positive $X_{t}$ and varying $\tan \beta$, this may be reduced slightly to $1.1 \mathrm{TeV}$.
11. S. Heinemeyer, W. Hollik, G. Weiglein, Eur. Phys. J. C 9, 343 (1999). https://doi.org/10.1007/s100529900006

12. S. Heinemeyer, W. Hollik, G. Weiglein, Phys. Lett. B 455, 179 (1999). https://doi.org/10.1016/S0370-2693(99)00417-7

13. G. Degrassi, P. Slavich, F. Zwirner, Nucl. Phys. B 611, 403 (2001). https://doi.org/10.1016/S0550-3213(01)00343-1

14. A. Brignole, G. Degrassi, P. Slavich, F. Zwirner, Nucl. Phys. B 631, 195 (2002). https://doi.org/10.1016/S0550-3213(02)00184-0

15. A. Dedes, G. Degrassi, P. Slavich, Nucl. Phys. B 672, 144 (2003). https://doi.org/10.1016/j.nuclphysb.2003.08.033

16. S. Heinemeyer, W. Hollik, H. Rzehak, G. Weiglein, Eur. Phys. J. C 39, 465 (2005). https://doi.org/10.1140/epjc/s2005-02112-6

17. S. Heinemeyer, W. Hollik, H. Rzehak, G. Weiglein, Phys. Lett. B 652, 300 (2007). https://doi.org/10.1016/j.physletb.2007.07.030

18. W. Hollik, S. Paßehr, JHEP 10, 171 (2014). https://doi.org/10. 1007/JHEP10(2014)171

19. S. Paßehr, G. Weiglein, Eur. Phys. J. C 78(3), 222 (2018). https:// doi.org/10.1140/epjc/s10052-018-5665-8

20. S.P. Martin, Phys. Rev. D 65, 116003 (2002). https://doi.org/10. 1103/PhysRevD.65.116003

21. S.P. Martin, Phys. Rev. D 66, 096001 (2002). https://doi.org/10. 1103/PhysRevD.66.096001

22. S.P. Martin, Phys. Rev. D 67, 095012 (2003). https://doi.org/10. 1103/PhysRevD.67.095012

23. A. Dedes, P. Slavich, Nucl. Phys. B 657, 333 (2003). https://doi. org/10.1016/S0550-3213(03)00173-1

24. A. Brignole, G. Degrassi, P. Slavich, F. Zwirner, Nucl. Phys. B 643 79 (2002). https://doi.org/10.1016/S0550-3213(02)00748-4

25. S.P. Martin, Phys. Rev. D 70, 016005 (2004). https://doi.org/10. 1103/PhysRevD.70.016005

26. S.P. Martin, Phys. Rev. D 71, 016012 (2005). https://doi.org/10. 1103/PhysRevD.71.016012

27. S.P. Martin, Phys. Rev. D 71, 116004 (2005). https://doi.org/10. 1103/PhysRevD.71.116004

28. S.P. Martin, Phys. Rev. D 75, 055005 (2007). https://doi.org/10. 1103/PhysRevD.75.055005

29. R.V. Harlander, P. Kant, L. Mihaila, M. Steinhauser, Phys. Rev, Lett. 100, 191602 (2008). https://doi.org/10.1103/PhysRevLett. 100.191602

30. R.V. Harlander, P. Kant, L. Mihaila, M. Steinhauser, Phys. Rev. Lett. 101, 039901 (2008). https://doi.org/10.1103/PhysRevLett. 101.039901

31. P. Kant, R.V. Harlander, L. Mihaila, M. Steinhauser, JHEP 08, 104 (2010). https://doi.org/10.1007/JHEP08(2010)104

32. S.P. Martin, Phys. Rev. D 96(9), 096005 (2017). https://doi.org/10 1103/PhysRevD.96.096005

33. The ATLAS collaboration, ATLAS-CONF-2017-020 (2017)

34. The ATLAS collaboration, ATLAS-CONF-2017-019 (2017)

35. M. Bicer et al., JHEP 01, 164 (2014). https://doi.org/10.1007/ JHEP01(2014)164

36. M. Dam, PoS EPS-HEP2015, 334 (2015)

37. P. Janot, PoS EPS-HEP2015, 333 (2015)

38. G.F. Giudice, A. Strumia, Nucl. Phys. B 858, 63 (2012). https:// doi.org/10.1016/j.nuclphysb.2012.01.001

39. P. Draper, G. Lee, C.E.M. Wagner, Phys. Rev. D 89(5), 055023 (2014). https://doi.org/10.1103/PhysRevD.89.055023

40. E. Bagnaschi, G.F. Giudice, P. Slavich, A. Strumia, JHEP 09, 092 (2014). https://doi.org/10.1007/JHEP09(2014)092

41. G. Lee, C.E.M. Wagner, Phys. Rev. D 92(7), 075032 (2015). https:// doi.org/10.1103/PhysRevD.92.075032

42. J. Pardo Vega, G. Villadoro, JHEP 07, 159 (2015). https://doi.org/ 10.1007/JHEP07(2015)159

43. B.C. Allanach, Comput. Phys. Commun. 143, 305 (2002). https:// doi.org/10.1016/S0010-4655(01)00460-X 
44. B.C. Allanach, A. Bednyakov, R. Ruiz de Austri, Comput. Phys. Commun. 189, 192 (2015). https://doi.org/10.1016/j.cpc.2014.12. 006

45. P. Athron, J.H. Park, D. Stöckinger, A. Voigt, Comput. Phys. Commun. 190, 139 (2015). https://doi.org/10.1016/j.cpc.2014.12.020

46. P. Athron, M. Bach, D. Harries, T. Kwasnitza, J.H. Park, D. Stöckinger, A. Voigt, J. Ziebell, Comput. Phys. Commun. 230, 145 (2018). https://doi.org/10.1016/j.cpc.2018.04.016

47. R.V. Harlander, J. Klappert, A. Voigt, Eur. Phys. J. C 77(12), 814 (2017). https://doi.org/10.1140/epjc/s10052-017-5368-6

48. H. Bahl, S. Heinemeyer, W. Hollik, G. Weiglein, Eur. Phys. J. C 78(1), 57 (2018). https://doi.org/10.1140/epjc/ s10052-018-5544-3

49. T. Hahn, S. Heinemeyer, W. Hollik, H. Rzehak, G. Weiglein, Phys. Rev. Lett. 112(14), 141801 (2014). https://doi.org/10.1103/ PhysRevLett.112.141801

50. H. Bahl, W. Hollik, Eur. Phys. J. C 76(9), 499 (2016). https://doi. org/10.1140/epjc/s10052-016-4354-8

51. P. Athron, J.H. Park, T. Steudtner, D. Stöckinger, A. Voigt, JHEP 01, 079 (2017). https://doi.org/10.1007/JHEP01(2017)079

52. L.V. Avdeev, M.Y. Kalmykov, Nucl. Phys. B 502, 419 (1997). https://doi.org/10.1016/S0550-3213(97)00404-5

53. A. Bednyakov, A. Onishchenko, V. Velizhanin, O. Veretin, Eur. Phys. J. C 29, 87 (2003). https://doi.org/10.1140/epjc/ s2003-01178-4

54. A. Bednyakov, D.I. Kazakov, A. Sheplyakov, Phys. Atom. Nucl. 71, 343 (2008). https://doi.org/10.1007/s11450-008-2015-6

55. G. Degrassi, S. Di Vita, J. Elias-Miro, J.R. Espinosa, G.F. Giudice, G. Isidori, A. Strumia, JHEP 08, 098 (2012). https://doi.org/10. 1007/JHEP08(2012)098

56. S.P. Martin, D.G. Robertson, Phys. Rev. D 90(7), 073010 (2014). https://doi.org/10.1103/PhysRevD.90.073010

57. E. Bagnaschi, J. Pardo Vega, P. Slavich, Eur. Phys. J. C 77(5), 334 (2017). https://doi.org/10.1140/epjc/s10052-017-4885-7
58. A.V. Bednyakov, A.F. Pikelner, V.N. Velizhanin, Nucl. Phys. B 875 , 552 (2013). https://doi.org/10.1016/j.nuclphysb.2013.07.015

59. D. Buttazzo, G. Degrassi, P.P. Giardino, G.F. Giudice, F. Sala, A. Salvio, A. Strumia, JHEP 12, 089 (2013). https://doi.org/10.1007/ JHEP12(2013)089

60. K.G. Chetyrkin, M. Steinhauser, Nucl. Phys. B 573, 617 (2000). https://doi.org/10.1016/S0550-3213(99)00784-1

61. K. Melnikov, T.V. Ritbergen, Phys. Lett. B 482, 99 (2000). https:// doi.org/10.1016/S0370-2693(00)00507-4

62. K.G. Chetyrkin, J.H. Kuhn, M. Steinhauser, Comput. Phys. Commun. 133, 43 (2000). https://doi.org/10.1016/ S0010-4655(00)00155-7

63. S.P. Martin, Phys. Rev. D 92(5), 054029 (2015). https://doi.org/10. 1103/PhysRevD.92.054029

64. K.G. Chetyrkin, M.F. Zoller, JHEP 06, 175 (2016). https://doi.org/ 10.1007/JHEP06(2016) 175

65. A.V. Bednyakov, A.F. Pikelner, Phys. Lett. B 762, 151 (2016). https://doi.org/10.1016/j.physletb.2016.09.007

66. E. Bagnaschi, A. Voigt, G. Weiglein, Higgs mass computation for hierarchical spectra in the MSSM using FlexibleSUSY (in preparation)

67. J. Braathen, M.D. Goodsell, M.E. Krauss, T. Opferkuch, F. Staub, Phys. Rev. D 97(1), 015011 (2018). https://doi.org/10.1103/ PhysRevD.97.015011

68. F. Staub, W. Porod, Eur. Phys. J. C 77(5), 338 (2017). https://doi. org/10.1140/epjc/s10052-017-4893-7

69. H. Bahl, W. Hollik (2018). arXiv:1805.00867

70. D.M. Pierce, J.A. Bagger, K.T. Matchev, R.J. Zhang, Nucl. Phys. B 491, 3 (1997). https://doi.org/10.1016/S0550-3213(96)00683-9

71. S.P. Martin, M.T. Vaughn, Phys. Rev. D 50, 2282 (1994). https:// doi.org/10.1103/PhysRevD.50.2282 [Erratum: Phys. Rev. D 78, 039903 (2008). https://doi.org/10.1103/PhysRevD.78.039903] 